\title{
MedienPädagogik
}

Zeitschrift für Theorie und Praxis der Medienbildung

\section{Veränderung von Einstellungen und Verhaltensdeterminanten mittels videobasierter Narrationen mit parallelen Erklärungen}

\author{
Christina Sick
}

\section{Zusammenfassung}

Einstellungen werden als Kompetenzdimension in Bildungsprozessen bisher kaum adressiert. Bei der gezielten Veränderung von Einstellungen durch (mediale) kommunikative Mittel (〈Persuasion〉) weist 〈Edutainment〉 einige Erfolge vor. Während argumentbasierte Persuasion eine intensive Auseinandersetzung mit Inhalten und Argumenten erfordert (〈Elaboration〉), setzt die Persuasion mit Geschichten darauf, dass sich Rezipientinnen und Rezipienten die gezeigten Verhaltensweisen und Einstellungen u.a. über die Erfahrung, in die Story einzutauchen, und Identifikation mit den Figuren aneignen. In der Praxis kombiniert Edutainment Film-Geschichten oft auch mit expliziten Informationen oder Argumenten. Da hier verschiedene Mechanismen greifen, soll in dieser experimentellen Studie geklärt werden, wie Medienangebote mit 〈hybridem〉 Inhaltsformat, die Geschichten und Argumente parallel darbieten, auf Einstellungen und andere Verhaltensdeterminanten wirken. Im Vergleich mit einem rein narrativen Format wird (1) die Wirksamkeit des hybriden Formats geprüft und anhand von Modellen Narrativer Persuasion erstmalig (2) dessen Wirkweise exploriert. Die Befunde zeigen, dass das hybride Format signifikant höhere Persuasionswirkung entfaltet in den Bereichen, die die zusätzlichen parallelen Erklärungen inhaltlich 
abdecken, und dass v.a. Personen mit schlechteren kognitiven Voraussetzungen (z.B. geringe Aufmerksamkeit, wenig Interesse,...) davon profitieren. Die Erkenntnisse zur Wirkweise synchron-hybrider Inhaltsformate geben wertvolle Hinweise zur Weiterentwicklung der Theorie in diesem Bereich. Zugleich besteht weiterer Forschungsbedarf zur komplexen Rolle von Widerstand und sozialem Druck im Persuasionsprozess.

\title{
Persuasion and the Change of Behavioral Determinants by means of Narrative Videos with Synchronous Explanations
}

\begin{abstract}
Attitudes are a dimension of competencies, but are hardly addressed ineducational processes. 〈Edutainment〉 is particularly successful in purposeful changing attitudes through means of (media) communication ((persuasion〉). While argument-based persuasion requires intensive examination of the information and arguments ('elaboration), persuasion with stories relies on recipients adapting the demonstrated behavior and attitudes through identification with the characters and the experience of being immersed in the story. In practice, edutainment often combines narrative films with explicit information or arguments. As these are different mechanisms, this experiential study aims at clarifying, how information with 〈hybrid〉 content format, i.e. a story with parallel arguments, affects attitudes and other behavioral determinants. The study examines (1) the persuasive effectiveness of the hybrid format in contrast to a purely narrative one and (2) the persuasion mechanisms by testing modells of narrative persuasion for the first time on information with hybrid content format. The results show that the hybrid format has a significantly higher persuasive effect, especially in the areas covered by the parallel explanations, and that participants with worse cognitive condition (e.g. low attention, little interest, ...) particularly benefit from the hybrid format. The findings concerning the persuasion mechanisms of the synchrounously hybrid format give valuable hints for further development of theory in this field. Yet there is still need for research concerning the complex role of resistance and social pressure in the persuasion process.
\end{abstract}




\section{Problemaufriss}

Kompetent ist eine Person nicht schon dann, wenn sie weiss, wie etwas Bestimmtes funktioniert oder wie sie sich in einer bestimmten Situation zu verhalten hätte. Die Person muss dieses Wissen auch anwenden können und v.a. wollen. Neben Wissen und Fertigkeiten stellen also Einstellungen - emotionale, motivationale und volitionale Haltungen - eine zentrale Kompetenzdimension dar (Euler und Hahn 2004). In der Sozialpsychologie bezeichnet «Einstellung» die latente Neigung einer Person, mit einem bestimmten Grad der Zustimmung oder Ablehnung wertend auf ein Objekt (Dinge, Menschen, Situationen, Ideen, Verhaltensweisen etc.) zu reagieren (Fishbein und Ajzen 2010). Nach dem Reasoned Action Approach (Fishbein und Ajzen 1975, 2010) sind die Einstellungen einer Person wichtige Verhaltensdeterminanten und zentrale Ansatzpunkte, um künftige Verhaltensweisen zu beeinflussen. Im Berufskontext sind Einstellungen also eine Basis professionellen Handelns. Deshalb ist es bei der beruflichen Aus-, Fort- und Weiterbildung angezeigt, neben Fachwissen und Fertigkeiten auch entsprechende Einstellungen gezielt zu adressieren und aufzubauen.

Beim Versuch, Einstellungen und andere Verhaltensdeterminanten mittels (medialer) Kommunikation (〈Persuasion〉) gezielt zu ändern, können Edutainment-Angebote einige Erfolge vorweisen. Die neuere Persuasionsforschung führt diese Persuasionseffekte v.a. auf das narrative Format zurück (Shen, Sheer, und Li 2015). 〈Klassische〉 Persuasionsansätze fokussieren demgegenüber v.a. Informationen im rhetorisch-argumentativen Format. In der Praxis kombinieren filmbasierte Edutainment-Angebote oft Geschichten mit rhetorischen Informationen oder Argumenten, z.B. via Epiloge oder synchron dargebotener Informationen, z.B. über einen Sprecher aus dem Off in der Sendung mit der Maus. Persuasionstheoretisch greifen bei der Verarbeitung von Geschichten andere kognitive Prozesse und Persuasionsmechanismen als bei rhetorisch-argumentativen Informationen (Bilandzic und Busselle 2013) - bei paralleler Verarbeitung führt dies u.U. zu Konkurrenz um kognitive Ressourcen. Allerdings fehlt in der Literatur bislang ein Modell zur parallelen kognitiven Verarbeitung, das Vorhersagen für die Persuasionswirkung erlaubt, und auch empirische Befunde hierzu sind sehr rar. Darum möchte die vorliegende Studie klären, wie videobasierte Informationsangebote mit «synchron-hybridem) 
Inhaltsformat, die also Geschichten und explizite Argumente parallel im Videoformat darbieten, auf Einstellungen und andere Verhaltensdeterminanten wirken. Die Studie verfolgt dabei zwei Ziele:

1. die Evidenzbasis zur persuasiven Wirksamkeit synchron-hybrider Inhaltsformate für die Gestaltung von Bildungsmaterialien zu erweitern und

2. Hinweise zur persuasiven Wirkweise synchron-hybrider Inhaltsformate zu finden. Dafür wurden erstmalig theoretische Wirkmechanismen Narrativer Persuasion an Informationen mit synchron-hybridem Inhaltsformat geprüft.

Im Folgenden werden zunächst das Objekt und die intendierte Richtung der Einstellungsänderung (persuasive Botschaft) und das verwendete Einstellungsmodell beschrieben. Daran schliesst sich ein Überblick der Forschungslage zur Persuasion mit Informationen im rhetorisch-argumentativen, im narrativen und im hybriden Format an. Sodann werden Studienaufbau und -ergebnisse dargestellt und abschliessend die Befunde mit Blick auf die Frage und die Ziele der Studie diskutiert.

\section{Einstellungsobjekt und persuasive Botschaft}

Einstellung(sänderung)en sind in verschiedenen Lebensbereichen relevant. Im Berufskontext von Lehrkräften spielen Einstellungen z.B. bei deren Erziehungsstil eine zentrale Rolle, da Lehrkräfte auch einen gesetzlichen Erziehungsauftrag haben. Der Erziehungsstil ist in konkreten, zeitlich und situationsübergreifend ziemlich konstanten Verhaltensweisen einer Person zu erkennen. (Domke 1991; Krohne und Hock 2010; Schneewind 1980; Sieland 1994) Das Erziehungsverhalten wird massgeblich beeinflusst durch die kognitive Dimension des Erziehungsstils (Schneewind 2008), die weniger ein Wissen, sondern eine bestimmte Erziehungshaltung meint, d.h. inwieweit jemand bestimmte Erziehungsgrundsätze eher positiv bewertet oder eher ablehnt.

Dabei ist es mit Blick auf das Kindswohl nicht beliebig, welchen Erziehungsstil eine Lehrkraft favorisiert. Die empirischen Befunde legen nahe, dass der autoritative Erziehungsstil im Vergleich zum autoritären, 
permissiven oder vernachlässigenden Stil zu favorisieren wäre: er begünstigt, dass Kinder und Jugendliche positivere Überzeugungen zu ihren Leistungen entwickeln und in der Folge bessere Schulleistungen (Steinberg, Elmen, und Mounts 1989), höhere Resilienz und emotionale Intelligenz haben (Fernandes 2016) und sich insgesamt eher zu «selbstbewussten, leistungsbereiten, gemeinschaftsfähigen und dabei auch noch lebensfrohen Personen entwickeln können» (Schneewind und Böhmert 2009, 16). Dieser Erziehungsstil folgt dem Grundsatz «Freiheit in Grenzen» mit jeweils hoch ausgeprägten Dimensionen «Wertschätzung und Unterstützung», «Fordern und Grenzen setzen» und «Gewähren und Fördern von Eigenständigkeit». (ebd.) Eine positive Einstellung zu diesem Grundsatz beeinflusst das konkrete Erziehungsverhalten einer Person und prägt deren Erziehungsstil positiv hin zum autoritativen Stil. Deshalb stellt dieser Grundsatz die inhaltliche Ausrichtung des Persuasionsversuchs dieser Studie dar, d.h. die persuasive Botschaft besteht in den Vorteilen des autoritativen Stils.

Die Fragestellung dieser Studie lässt sich nun wie folgt präzisieren: Wie wirken Informationen mit synchron-hybridem Inhaltsformat auf Einstellungen und andere Verhaltensdeterminanten Lehramtsstudierender zu autoritativem Erziehungsverhalten?

\section{Einstellungen: Reasoned Action Approach (RAA)}

$\mathrm{Da}$ die Erziehungsstilforschung kein Einstellungskonzept vorhält, das anschlussfähig ist an die psychologische Einstellungsforschung, wird das Einstellungsobjekt hier im Sinne des Reasoned Action Approach (RAA; Fishbein und Ajzen 2010) modelliert und operationalisiert. Dieser ist in der Einstellungsforschung gut etabliert und stellt passend für das Objekt «Erziehungsstile» auf eine enge Beziehung von Einstellung und Verhalten ab, die sich daraus ergibt, dass Einstellungen nicht generell, sondern stets in Bezug auf ein bestimmtes Verhalten als attitude toward the behavior formuliert werden. Das Einstellungsobjekt soll mit dem Zielverhalten in time, target, action und context bestmöglich korrespondieren. Diese enge Bindung an ein Zielverhalten führt zu einer weiteren Besonderheit des Ansatzes: zur Einstellung kommen weitere Verhaltensdeterminanten hinzu, da die Einstellung zur Verhaltensvorhersage allein nicht ausreicht. Das aktuelle 
RAA-Modell, das integrative model of behavior prediction (IM; Fishbein 2008, Fishbein und Ajzen 2010), führt neben der Einstellungs- eine Norm- und eine Kontrolldeterminante, die zusammen die Verhaltensintention prägen und sich der Erwartungs-mal-Wert-Logik folgend auf dahinterliegende Überzeugungen stützen, die zudem von Hintergrundfaktoren beeinflusst werden (s. Abbildung 1).

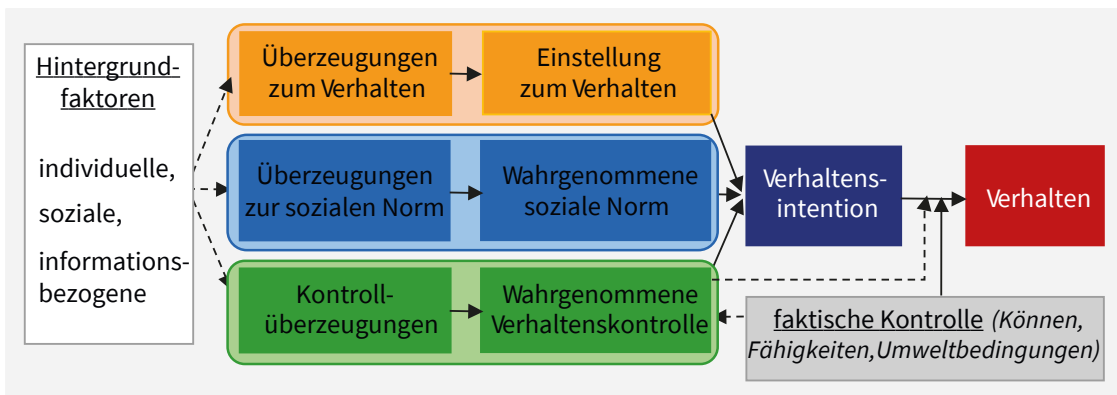

Abb. 1.: Das Integrative Modell der Verhaltensvorhersage (nach Fishbein und Ajzen 2010).

Für die gezielte Einstellungsänderung hält die Forschung eigene Theorien bereit, die mehrheitlich die Persuasionswirkung vom Inhaltsformat (rhetorisch-argumentativ oder narrativ), weniger von der Mediengestalt her modellieren. Der besseren Theorielage wegen stellt die vorliegende Studie auf das Inhaltsformat als erklärenden Faktor ab statt auf Merkmale des Mediums Film. Da keine theoretischen Modelle zur Persuasion mit Informationen mit hybridem Inhaltsformat vorliegen, wird auf Theorien der argumentbasierten und der narrativen Persuasion zurückgegriffen sowie auf ein Modell des Lernens mit Informationen mit hybridem Inhaltsformat.

\section{Elaboration als Erfolgsfaktor der rhetorisch-argu- mentbasierten Persuasion}

Unter den etablierten Persuasionstheorien werden die Zwei-Prozess-Modelle am stärksten rezipiert, v.a. das Elaboration Likelihood Model (ELM; z.B. Cacioppo und Petty 1984, Petty und Cacioppo 1986). Kern dieses Modells ist die Elaboration, also die gedankliche Auseinandersetzung mit dem Gegenstand der dargebotenen persuasiven Informationen, die i.d.R. von den 
Argumenten der Botschaft hervorgerufen, aber auch von «non-message factors» beeinflusst werden können, z.B. Attraktivität, Expertise oder Glaubwürdigkeit der Quelle (Petty und Wegener 1999). Hohe Elaboration «involves paying careful attention to the relevant information in the message, relating that information to previous knowledge stored in memory (e.g., is the message consistent [...] with other facts that I know?), and generating new implications of the information» (Petty, Cacioppo, Strathman, und Priester 2005, 84).

Rezipientinnen und Rezipienten beziehen hier sowohl Informationen des Inhalts (Argumentgüte) als auch des unmittelbaren Kontextes in ihre gedankliche Prüfung mit ein (Petty und Cacioppo 1984).

«At the low end of the elaboration continuum, information scrutiny is reduced [...] e.g., examining less information than when elaboration is high or examining the same information less carefully» (Petty und Wegener 1999, 42 ).

Elaboration ist der kritische Faktor der argumentbasierten Persuasion. Kommt die Einstellungsänderung durch hohe Elaboration zustande, ist diese nachhaltig und stabil (Petty 1997); entsteht sie auf dem kognitiv leichteren Weg der Prüfung peripherer Merkmale («non-message factors»), wird die neue Einstellung schlechter erinnert, ist zeitlich relativ instabil und anfälliger für Infragestellung (Cacioppo und Petty 1984). Drei Bedingungen erhöhen laut ELM die Elaborationswahrscheinlichkeit:

- hohe Fähigkeit einer Person zur Elaboration, d.h. hohe individuelle Intelligenz und Vorwissen sowie geringe situative Ablenkung,

- hohe Motivation, gespeist aus der intrinsischen Freude an intensivem Nachdenken (Kognitionsbedürfnis) und dem Wunsch einer Person, korrekte Urteile zu treffen, was wiederum gefördert wird von

- hohem Involvement, d.h. hohe persönliche Relevanz des Einstellungsobjekts. Neben der Höhe der Elaboration zählt aber auch ihre Valenz: Machen sich Rezipientinnen und Rezipienten bei hoher Elaboration mehr negative als positive Gedanken zur vertretenen Position, wirkt intensive gedankliche Auseinandersetzung kontraproduktiv und verstärkt die bisherige Einstellung. Elaboration ist mit Blick auf den Persuasionserfolg also ambivalent. 


\section{Absorption und die Reduktion von Widerstand und Elaboration als Mechanismen Narrativer Persuasion}

Die Persuasionswirkung von Geschichten lässt sich mit klassischen Persuasionstheorien nur unzureichend beschreiben; sie baut auf andere Mechanismen als die mit rhetorischen Informationen. (Green und Brock 2000; Slater und Rouner 2002) Diese Mechanismen werden im Folgenden skizziert und in der Studie erstmalig mit Informationen geprüft, die Inhalte im narrativen und argumentativen Format verbinden.

1. Die Modelle Narrativer Persuasion stützen sich massgeblich auf die Qualität des psychischen Erlebens bei der Rezeption von Geschichten. Die verschiedenen Konstrukte dieses Rezeptionserlebens kreisen darum, dass Rezipientinnen und Rezipienten in Geschichten hineingezogen werden und sich darin verlieren (Nell 1988). Transportation und Narrative Engagement (Busselle und Bilandzic 2009) seien hier als die wichtigsten genannt. Daneben spielt die Beziehung der Rezipientinnen und Rezipienten zu den Figuren bzw. Interaktion mit diesen eine zentrale Rolle: gemeint ist hier z.B. die Identifikation mit diesen (Cohen 2001) oder die wahrgenommene Ähnlichkeit. Hohes Narrative Engagement und hohe Identifikation werden ausgelöst durch Merkmale der Narration, der Rezipientinnen und Rezipienten sowie des Kontextes. Narrative Engagement und Identifikation befördern nachweislich die Veränderung von Einstellungen und z.T. anderen Verhaltensdeterminanten (u.a. Busselle und Bilandzic 2009; De Graaf, Hoeken, Sanders und Beentjes 2012; Green und Brock 2002; Moyer-Gusé 2008; van Leeuwen, Putte, Renes und Leeuwis 2017), u.a. weil über Identifikation sowie das intensive kognitive und emotionale Eintauchen in die Geschichten und die dabei entstehenden lebhaften Bildvorstellungen die vertretenen Werte und Einstellungen der Hauptfiguren verstanden und übernommen werden.

2. Ein anderer Persuasionsmechanismus bei der Rezeption von Geschichten ist die Reduktion von Widerstand, einem Haupthindernis bei argumentbasierter Persuasion. Ein Grund dafür ist, dass die persuasive Botschaft in Geschichten implizit ist, was es Rezipientinnen und Rezipienten schwer macht, Gegenargumente aufzubringen, da sie zuerst den vertretenen Standpunkt erkennen und benennen müssen. (Dal 
Cin, Zanna und Fong 2004; Slater und Rouner 2002) Ausserdem sind Rezipientinnen und Rezipienten generell offen für die Inhalte der Geschichte, da sie diese als Unterhaltung sehen, und erwarten darum kaum eine Persuasionsabsicht. (Petty und Cacioppo 1986; Hoeken und Fikkers 2014) Die Reduktion von Widerstand wird ferner auf begrenzte kognitive Verarbeitungskapazitäten zurückgeführt: Da die Verarbeitung narrativer Informationen als eigener Prozess kognitiver Informationsverarbeitung verstanden wird, der v.a. die Produktion mentaler Modelle der rezipierten Geschichte meint (Graesser, Olde, und Klettke 2002), bleibt bei Ressourcenknappheit dank Primat der narrativen Verarbeitung wenig Kapazität für andere Gedanken (Lang 2000), sodass Rezipientinnen und Rezipienten bei hoher Transportation zu weniger Counterarguing neigten (Green und Brock 2000) bzw. weniger stark eine Persuasionsabsicht vermuteten (Moyer-Gusé und Nabi 2010).

3. Aus demselben Grund wird zuweilen auch die Position postuliert, dass hohe Absorption sogar generell die Elaboration der Inhalte der Story unterdrücke, wie z.B. Dal Cin, Zanna und Fong (2004) meinen.

\section{Verarbeitung von Informationen mit synchron-hyb- riden Inhaltsformat}

Zur Frage nach der Persuasionswirkung und -wirkweise von Informationen mit synchron-hybridem Inhaltsformat gibt es keine eigenen Modelle, aber es können Anleihen z.B. beim Capacity Model of Comprehension of Educational Content on Television (Fisch 2000) gemacht werden, das sich auf das Lernen edukativer Inhalte in narrativen Fernsehsendungen bezieht. Das Modell geht von zwei distinkten Verarbeitungsprozessen für narrative und edukative Informationen aus, die beide nötig sind zur Verarbeitung von hybriden Informationen. Treten sie synchron auf, werden Tiefe und Umfang der Verarbeitungsprozesse durch die Begrenzung der Kapazitäten des Arbeitsgedächtnisses eingeschränkt. Tiefe und Umfang der Verarbeitung edukativer Inhalte hängen dabei v.a. von der Ressourcenverteilung auf die simultan geforderten Prozesse ab. Dabei spielen mehrere Faktoren eine Rolle: der Vorrang narrativer Verarbeitung, die situative Verfügbarkeit kognitiver Ressourcen (also wie anspruchsvoll die Geschichte ist), ob die 
Ressourcenverteilung absichtsvoll erfolgt (also Massnahmen vorhanden sind, die die edukativen Inhalte salienter machen) und v.a. wie gross die sog. Distanz zwischen narrativen und edukativen Inhalten ist.

«[W]hen the distance between narrative and educational content is small [...], then the two parallel processes become complementary rather than competitive, and comprehension is likely to be strengthened.» (Fisch 2000, 66)

Das Capacity-Modell (Fisch 2000) bietet mithin Hinweise, unter welchen Bedingungen narrative und nicht-narrative Informationen synchron verarbeitet werden können. Wie die parallele Verarbeitung auf Einstellungen und andere Verhaltensdeterminanten wirkt, lässt sich aber daraus nicht ableiten, weshalb dies durch eine empirische Untersuchung ermittelt werden soll.

\section{Studiendesign}

Die Studie untersucht, wie Informationen mit synchron-hybridem Inhaltsformat auf Einstellungen und andere Verhaltensdeterminanten Lehramtsstudierender zu autoritativem Erziehungsverhalten wirken, und zwar in zweifacher Hinsicht: mit Blick auf die persuasive (1) Wirksamkeit und (2) Wirkweise synchron-hybrider Inhaltsformate.

In einer experimentellen Studie mit 141 Lehramtsstudierenden der $\mathrm{PH}$ Weingarten sah von den vier Untersuchungsgruppen Treatmentgruppe 1 eine narrative Filmszene zur Erziehungssituation «Ich kann das nicht!» (Schneewind 2008), Treatmentgruppe 2 dieselbe Geschichte mit zusätzlichen parallelen Erklärungen eines Sprechers aus dem Off (synchron-hybrides Format), die auf der Einstellungsebene ansetzten und Vorteile des gezeigten autoritativen Erziehungsverhaltens nannten. Zwei Kontrollgruppen erhielten ein Video anderen Inhalts; hier variierte die Reihenfolge der Aufgaben, um eine Verzerrung durch die Messinstrumente zu prüfen.

Als abhängige Variablen der persuasiven Wirkung fungieren die RAAKomponenten (Fishbein und Ajzen 2010), die persuasive Wirkung (Wirksamkeit und Wirkweise) wird also hinsichtlich folgender Variablen erfasst: Verhaltensintention, Einstellungs-, Kontroll- und Normdeterminante des 
IM samt der jeweils dazugehörigen Überzeugungen zu autoritativem Erziehungsverhalten, sowie zusätzlich die Einstellungen zu den alternativen Verhaltensweisen (autoritär, permissiv, autoritativ) mit konkreterer Formulierung. Die Messung erfolgte vor und nach dem Treatment (prä-postDesign).

Um die persuasive Wirkweise von Informationen mit synchron-hybridem Inhaltsformat zu erkunden, wurden erstmalig zentrale Wirkmechanismen narrativer und argumentbasierter Persuasion (Wirkung von Rezeptionserleben und Identifikation, Widerstandsreduktion, Wirkung der Elaboration) an Informationen mit synchron-hybridem Inhaltsformat getestet, unter Berücksichtigung der Capacity-Modell-Postulate.

Dafür wurden zusätzliche Variablen erhoben, die die Wirkung der Videos auf die Einstellungen und andere Verhaltensdeterminanten theoretisch beeinflussen:

1. An Hintergrundfaktoren wurden erhoben: Geschlecht, Alter, Vorwissen, vergangenes Verhalten, Involvement (d.h. subjektive Relevanz des Themas), Stadt-Land (Heimatortgrösse), Studiengang (Grundschule, Sekundarstufe I), Schulpraxiserfahrung, Fachsemesterzahl, Bildungsgrad, Migrationshintergrund sowie das Vorhandensein eigener Kinder, wobei die letzten drei Variablen zu wenig Varianz aufwiesen und darum aus weiteren Berechnungen ausgeschlossen wurden.

2. Ausserdem wurden zwischen der Videorezeption und der zweiten $A b-$ frage der Outcome-Variablen folgende die Persuasionswirkung evtl. vermittelnde Variablen erfasst: als Variablen des Rezeptionserlebens und der Interaktion mit den Figuren die Identifikation (Skala von Cohen 2001) und das Narrative Engagement (mit den vier Faktoren Narratives Verstehen, Emotionale Beteiligung, Narrative Präsenz, Aufmerksamkeitsfokus; Skala von Busselle und Bilandzic 2009); als Widerstandsvariablen die Reaktanz (Skala von Dillard und Shen 2005) und wahrgenommene Persuasionsabsicht (Skala von Moyer-Gusé, Jain und Chung 2012 bzw. Petty und Cacioppo 1979); als Variablen der gedankliche Auseinandersetzung die rezeptionsbegleitende Reflexion und die post-rezeptive Elaboration (per «thought-listing»). 
Tabelle 1 zeigt das Variablenmodell der Studie. Die Fragen und Hypothesen der Studie sowie die Auswertungsmethoden werden bei der Ergebnisdarstellung aufgeführt.

\begin{tabular}{|c|c|c|c|c|}
\hline $\begin{array}{l}\text { Hintergrund- } \\
\text { faktoren }\end{array}$ & $\begin{array}{l}\text { Persuasionsoutcome } \\
\text { zum Messzeitpunkt z1 } \\
\text { (vor dem Treatment) }\end{array}$ & $\begin{array}{l}\text { Untersuchungs- } \\
\text { merkmal } \\
\text { des Treatments }\end{array}$ & $\begin{array}{l}\text { Kognitiv-affektive } \\
\text { Reaktionen bei der } \\
\text { Informationsver- } \\
\text { arbeitung }\end{array}$ & $\begin{array}{l}\text { Persuasionsoutcome } \\
\text { zum Messzeitpunkt } \underline{22} \\
\text { (nach dem Treatment) }\end{array}$ \\
\hline $\begin{array}{l}\text { Vergangenes } \\
\text { Verhalten }\end{array}$ & Verhaltensintention & Inhaltsformat & & Verhaltensintention \\
\hline \multirow{2}{*}{$\begin{array}{l}\text { Schulpraxis- } \\
\text { erfahrung }\end{array}$} & \multirow{3}{*}{$\begin{array}{l}\text { Einstellung zum } \\
\text { (Ziel)Verhalten }\end{array}$} & \multirow{3}{*}{$\begin{array}{l}\text { synchron-hybrid } \\
\text { vs. } \\
\text { rein narrativ }\end{array}$} & \multirow{3}{*}{ 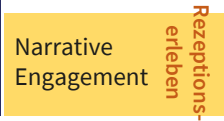 } & \multirow{3}{*}{$\begin{array}{l}\text { Einstellung zum } \\
\text { (Ziel)Verhalten }\end{array}$} \\
\hline & & & & \\
\hline Vorwissen & & & & \\
\hline Involvement & \multirow{2}{*}{$\begin{array}{l}\text { Wahrgenommene } \\
\text { soziale Norm }\end{array}$} & & \multirow{4}{*}{ 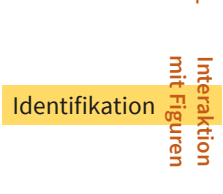 } & \multirow{2}{*}{$\begin{array}{l}\text { Wahrgenommene } \\
\text { soziale Norm }\end{array}$} \\
\hline Geschlecht & & & & \\
\hline Alter & \multirow{2}{*}{ 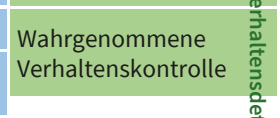 } & & & \multirow{2}{*}{$\begin{array}{ll}\text { Wahrgenommene } & \frac{\frac{0}{7}}{\frac{0}{0}} \\
\text { Verhaltenskontrolle } & \frac{0}{\overline{0}} \\
\frac{0}{0}\end{array}$} \\
\hline $\begin{array}{l}\text { Heimatort- } \\
\text { grösse }\end{array}$ & & & & \\
\hline $\begin{array}{l}\text { Fachse- } \\
\text { mester }\end{array}$ & $\begin{array}{l}\text { Überzeugungen } \\
\text { zum (Ziel)Verhalten }\end{array}$ & & \multirow{3}{*}{$\begin{array}{l}\text { Wahrgenom- } \\
\text { mene Persuati- } \\
\text { onsabsicht } \\
\text { Reaktanz }\end{array}$} & $\begin{array}{l}\text { Überzeugungen } \\
\text { zum (Ziel)Verhalten }\end{array}$ \\
\hline \multirow{2}{*}{$\begin{array}{l}\text { Studiengang } \\
\text { GS - Sek I }\end{array}$} & \multirow{2}{*}{$\begin{array}{l}\text { Überzeugungen zur } \\
\text { sozialen Norm }\end{array}$} & & & \multirow{2}{*}{$\begin{array}{l}\text { Überzeugungen zur } \\
\text { sozialen Norm }\end{array}$} \\
\hline & & & & \\
\hline $\begin{array}{l}\text { Bildungs- } \\
\text { grad* }^{*}\end{array}$ & $\begin{array}{l}\text { Kontrollüberzeu- } \\
\text { gungen }\end{array}$ & & & $\begin{array}{l}\text { Kontrollüberzeu- } \\
\text { gungen }\end{array}$ \\
\hline $\begin{array}{l}\text { Migrations- } \\
\text { hintergrund* }\end{array}$ & \multirow{2}{*}{$\begin{array}{l}\text { Einstellung zu autorita } \\
\text { tivem Verhalten }\end{array}$} & & \multirow{2}{*}{$\begin{array}{l}\text { postrezeptive } \\
\text { Reflexion }\end{array}$} & \multirow{2}{*}{$\begin{array}{l}\text { Einstellung zu autorita } \\
\text { tivem Verhalten }\end{array}$} \\
\hline \multirow{3}{*}{$\begin{array}{l}\text { Eigene } \\
\text { Kinder* }\end{array}$} & & & & \\
\hline & \multirow{2}{*}{$\begin{array}{l}\text { Einstellung zu autori- } \\
\text { tärem Verhalten } \\
\text { Einstellung zu permis- } \\
\text { sivem Verhalten }\end{array}$} & & \multirow{2}{*}{ 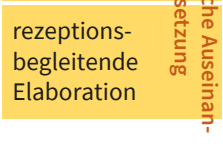 } & $\begin{array}{l}\text { Einstellung zu autori- } \\
\text { tärem Verhalten }\end{array}$ \\
\hline & & & & 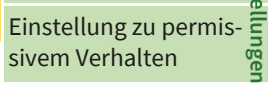 \\
\hline
\end{tabular}

Tab. 1.: Variablenmodell der Studie. Anmerkung. * zu vernachlässigen, da zu geringer Anteil in Stichprobe, der vom Mainstream darin abweicht.

\section{Ergebnisse}

Die Studierenden befanden sich bei der Erhebung fast alle in bzw. am Anfang ihrer Pflichtpraktika, waren 18 bis 38 Jahre alt $(M=21.96, S D=3.27)$ und der Geschlechterverteilung der PH Weingarten entsprechend zu 77,3\% weiblich und $\mathrm{zu}$ 22,7\% männlich (Studierendensekretariat PH Weingarten 2016), hatten zu 83,3\% die allgemeine Hochschulreife als höchsten Bildungsgrad und im Schnitt 54 Tage Schulpraxiserfahrung (knapp ein Drittel noch keine), stammten mehrheitlich $(78,2 \%)$ vom Lande und studierten 
ca. je zur Hälfte Lehramt für Sekundarstufe I und Grundschule. Das Involvement der Probandinnen und Probanden ( $\mathrm{N}=141)$ in die Thematik Erziehungsstile war sehr hoch $(\mathrm{M}=5.81, \mathrm{SD}=.76)$, aber die Einschätzung des eigenen Vorwissens zum Thema eher niedrig ( $\mathrm{M}=3.74, \mathrm{SD}=1.17)$. Mehr als die Hälfte $(58,2 \%)$ derer, die eine vergleichbare Situationen erlebt zu haben meinten, gab an, ihr eigenes vergangenes Verhalten in dieser Situation hätte «ziemlich genauso» oder sogar «genauso» wie das beschriebene autoritative Zielverhalten ausgesehen.

\subsection{Befunde zur persuasiven Wirksamkeit des synchron- hybriden Inhaltsformats}

Die Forschungsfrage nach der persuasiven Wirkung von Informationen mit synchron-hybridem Inhaltsformat ist auf zwei Ziele ausgerichtet: Exploration der (1) persuasiven Wirksamkeit und (2) persuasiven Wirkweise videobasierter Narrationen mit parallelen argumentativen Erklärungen. Untersuchungsfrage 1 und 2 adressieren Ziel 1.

Untersuchungsfrage 1 analysierte, ob die eingesetzten Treatments ( $\mathrm{T} 1$, T2) im Vergleich zu den beiden Kontrollgruppen mit Kontrollvideo (K1, K2) eine intendierte persuasive Wirkung entfalteten. Aufgrund der positiven Befunde Narrativer Persuasion wurde für T1 und T2 eine positive Wirkung bei mind. einer Outcome-Variablen angenommen $(\mathrm{Hl})$. In Ermangelung von Äquivalenzgrenzen zu den Outcome-Variablen wurde deren BaselineGleichheit bei allen vier Gruppen zum Zeitpunkt zl statt mit Äquivalenztests mittels einfaktorieller ANOVA ohne Messwiederholung festgestellt. Auf dieser Basis konnten t-Tests bei allen vier Gruppen mit abhängigen Stichproben für alle Outcome-Variablen $\mathrm{H}$ bestätigen: beide Treatments zeigten signifikante intendierte Änderungen (i.d.R. Steigerung) von Zeitpunkt zl nach z2 und waren also wie intendiert wirksam, wobei die Kontrollvideos bei $\mathrm{K} 1$ und $\mathrm{K} 2$ ohne Wirkung blieben und reaktive Effekte des «thought-listings» ausgeschlossen wurden. Tabelle 2 listet die deskriptiven Outcomewerte aller Gruppen und die der Veränderungen über die Zeit im within-Test zwischen Messzeitpunkt zl (vor dem Treatment) und Messzeitpunkt z2 (nach dem Treatment). 


\begin{tabular}{|c|c|c|c|c|c|c|c|c|c|c|}
\hline & \multicolumn{3}{|c|}{ Messzeitpunkt z1 } & \multicolumn{3}{|c|}{ Messzeitpunkt z2 } & \multicolumn{3}{|c|}{ Differenz } \\
\hline & & $\mathrm{N}$ & M & SD & $\mathrm{N}$ & M & SD & $\mathrm{t}$ & $\mathrm{df}$ & $\mathrm{p}$ \\
\hline \multirow[t]{2}{*}{ Verhaltensintention } & T1 & 35 & 5.51 & 1.15 & 35 & 6.09 & .95 & .26 & 34 & .003 \\
\hline & $\mathrm{T} 2$ & 35 & & 97 & 34 & & 0.73 & 2.43 & 33 & .021 \\
\hline \multirow{2}{*}{$\begin{array}{l}\text { Einstellung zum } \\
\text { Verhalten }\end{array}$} & $\mathrm{T} 1$ & 35 & & & 35 & & & & & \\
\hline & T2 & 35 & & & 35 & & & & 34 & \\
\hline \multirow{2}{*}{$\begin{array}{l}\text { Wahrgenommene } \\
\text { soziale Norm }\end{array}$} & $\mathrm{T} 1$ & 35 & & & 35 & & & .07 & 34 & .046 \\
\hline & T2 & 35 & & & 35 & & & & & \\
\hline \multirow{2}{*}{$\begin{array}{l}\text { Wahrgenommene } \\
\text { Verhaltenskontrolle }\end{array}$} & $\mathrm{T} 1$ & 35 & & & 35 & & & & & \\
\hline & $\mathrm{T} 2$ & 35 & 6.80 & 35 & 35 & 1 & 7 & & 34 & .000 \\
\hline \multirow{2}{*}{$\begin{array}{l}\text { Überzeugungen } \\
\text { zum Verhalten }\end{array}$} & T1 & 35 & & & 35 & & & & 34 & 000 \\
\hline & $\mathrm{T} 2$ & 35 & & 31.76 & 35 & & & & & \\
\hline \multirow{2}{*}{$\begin{array}{l}\text { Überzeugungen zur } \\
\text { sozialen Norm }\end{array}$} & $\mathrm{T} 1$ & 31 & & 36.23 & 34 & & & & & .011 \\
\hline & T2 & 30 & 73 & .03 & 31 & & 9 & & 9 & .872 \\
\hline \multirow{2}{*}{$\begin{array}{l}\text { Kontrollüberzeu- } \\
\text { gungen }\end{array}$} & T1 & 35 & & & 35 & & & & & \\
\hline & T2 & 34 & 0 & .25 & 35 & & 1 & & & .052 \\
\hline \multirow{2}{*}{$\begin{array}{l}\text { Einstellung zu auto- } \\
\text { ritativem Verhalten }\end{array}$} & $\mathrm{T} 1$ & 35 & 919 & & 35 & & 79 & & 34 & 1.000 \\
\hline & $\mathrm{T} 2$ & 35 & 19.34 & & 35 & & & & 34 & .101 \\
\hline \multirow{2}{*}{$\begin{array}{l}\text { Einstellung zu auto- } \\
\text { toritärem Verhalten }\end{array}$} & $\mathrm{T} 1$ & 35 & & & 35 & & & & 34 & .743 \\
\hline & $\mathrm{T} 2$ & 35 & & & 35 & & & & 34 & .144 \\
\hline \multirow{2}{*}{$\begin{array}{l}\text { Einstellung zu per- } \\
\text { missivem Verhalten }\end{array}$} & $\mathrm{T} 1$ & 35 & & & 35 & & 5.11 & 1.22 & 34 & .231 \\
\hline & $\mathrm{T} 2$ & 35 & 11.29 & 3.37 & 35 & 9.17 & 4.34 & -2.88 & 34 & .007 \\
\hline
\end{tabular}

Tab. 2.: Änderung der Outcomevariablen der Treatmentgruppen T1 und T2 (prä/ post-Test). ${ }^{*}$ Anmerkung. Fett gedruckt $=p<.05$, Fett kursiv gedruckt $=$ $\mathrm{p}<.10$.

T1- und T2-Rezipientinnen und -Rezipienten neigten signifikant mehr als vor den Videos dem autoritativen Erziehungsverhalten zu (Einstellung zum Verhalten), glaubten mehr an die positiven Effekte (Überzeugungen zum Verhalten), wollten sich eher so verhalten (Verhaltensintention) und glaubten stärker als davor, es zu können (wahrgenommene Verhaltenskontrolle, Kontrollüberzeugungen knapp signifikant). Nur die Rezipientinnen und Rezipienten des rein narrativen Formats ( $\mathrm{T} 1)$ nahmen eine signifikant höhere soziale Norm bzgl. des autoritativen Zielverhaltens wahr und glaubten eher, relevante Bezugspersonen würden dieses Verhalten unterstützen und selbst tun (Überzeugungen zur sozialen Norm). Dafür sank allein beim hybriden Format (T2) die Zustimmung zur permissiven Verhaltensweise (Einstellung zu permissivem Verhalten). Die ausgebliebene Veränderung 
bei Einstellungen zu den anderen alternativen Verhaltensweisen lässt sich Deckeneffekten zuschreiben.

Untersuchungsfrage 2 prüfte mittels t-Tests mit unabhängigen Stichproben zum Zeitpunkt z2, inwiefern sich das narrative ( $\mathrm{T} 1)$ und das hybride Inhaltsformat (T2) in ihrer Wirkung auf die Outcome-Variablen unterscheiden. Wegen unterschiedlicher kognitiver Verarbeitungsprozesse wurde ein Unterschied angenommen ( $\mathrm{H} 2 \mathrm{a})$ und höhere Werte bei den Überzeugungen zum Verhalten beim hybriden Format, da die rhetorischen Informationen inhaltlich hier ansetzten (H2b). Die Befunde bestätigten $\mathrm{H} 2 \mathrm{a}$ und H2b: Rezipientinnen und Rezipienten des hybriden Formats glaubten signifikant stärker an die positiven Effekte des autoritativen Zielverhaltens (Überzeugungen zum Verhalten) als die des rein narrativen, $t(68)=2.876, p$ $=.003, \Delta=0.69$ (mittlere, fast grosse Effektstärke).

Hinzu kommen weitere, marginal signifikante Unterschiede bei der Einstellung zu autoritärem Verhalten, $t(68)=-1.926, p=.058, \Delta=-0.46$, und der zu permissivem Verhalten, $t(68)=1.762, p=.082, \Delta=-0.42$, bei denen wieder das synchron-hybride Treatment (T2) besser abschnitt. Diese Werte sind wegen der engen Verbindung zu den Überzeugungen zum Verhalten als Indizien für weitere Unterschiede zu sehen ( $\mathrm{H} 2 \mathrm{a})$.

Ergänzende Explorationen versuchten mitt-Tests zu ergründen, ob sich die beiden Inhaltsformate in ihrer Wirkung auf den Outcome unterschieden, wenn Variablen des Outcomes vor dem Treatment («Voreinstellung») und des persönlichen Hintergrunds niedrig bzw. hoch ausgeprägt waren. Dies bestätigte sich bei diversen Hintergrundbedingungen, wobei das hybride Format (T2) stets besser abschnitt, wenn Rezipientinnen und Rezipienten jung und am Studienbeginn waren, wenig Praxiserfahrung und keine Erfahrung mit der Situation hatten, vom Lande stammten, Grundschullehramt studierten und das Thema wenig Relevanz für sie hatte (Involvement). Wich das eigene Verhalten in der Vergangenheit eher vom Zielverhalten ab, schnitt das hybride Format ebenfalls besser ab. Geschlecht blieb ohne Effekt; hohes Vorwissen verstärkte die positive Wirkung des hybriden Formats auf die Überzeugungen zum Verhalten. Bei den übrigen Hintergrundbedingungen erzielten beide Treatments die gleiche Wirkung. Auch bei mehreren Bedingungen der «Voreinstellung» zeigten sich stärker positive Effekte des hybriden Formats (T2), i.d.R. wenn die Werte bereits 
hoch waren. Allerdings erwies sich das rein narrative Format ( $\mathrm{Tl}$ ) in einem Fall als wirksamer, nämlich auf die wahrgenommene soziale Norm, wenn Rezipientinnen und Rezipienten vor dem Treatment schon von den Vorzügen des Zielverhaltens überzeugt waren (Überzeugungen zum Verhalten) oder meinten, dieses Verhalten selbst tun zu können (wahrgenommene Verhaltenskontrolle).

\subsection{Befunde zur Wirkweise I: Einfluss des hybriden Formats auf Indikatoren der Informationsverarbeitung}

Um Hinweise auf die persuasive Wirkweise des synchron-hybriden Inhaltsformats zu erhalten (Ziel 2), wurden theoretisch beschriebene Wirkmechanismen Narrativer Persuasion erstmalig mit einem hybridem Format getestet. Dafür wurden in Untersuchungsfrage 3 - 5 Effekte des Inhaltsformats auf Variablen des Rezeptionserlebens und der Identifikation mit den Figuren (Frage 3), auf die Widerstandsreduktion (Frage 4) und die Reduktion von gedanklicher Auseinandersetzung mit der persuasiven Botschaft (Frage 5) untersucht und die Wirkung von Hintergrundfaktoren kontrolliert.

Untersuchungsfrage 3 überprüfte mittels Äquivalenztest, ob das Inhaltsformat das Narrative Engagement (NE) nicht beeinflusst. Da die Erklärungen im Reizmaterial eine geringe Distanz zur Geschichte haben, wird von keiner Ressourcenkonkurrenz (Fisch 2000) und ergo von keinem Unterschied im NE-Level beider Gruppen ausgegangen (H3a), aber evtl. bei einzelnen NE-Dimensionen (H3b). So wurde mittels t-Tests geprüft, ob bei mind. einer NE-Dimension zwischen beiden Inhaltsformaten ein Unterschied auftritt und ob die Identifikation beim hybriden Format niedriger ausfällt, da laut Cohen (2001) nicht-narrative Formate die Identifikation reduzieren können, was auch für das hybride Format zutreffen könnte (H3c). Wie H3a annahm waren die Treatments $\mathrm{Tl}(\mathrm{N}=35, \mathrm{M}=5.27, \mathrm{SD}=.81)$ und T2 ( $\mathrm{N}=35, \mathrm{M}=5.29, \mathrm{SD}=.94)$ bzgl. Narrative Engagement äquivalent (Grenzen \pm 0.95, $-0.36 ; 0.34$ 90\%-CI, t(68) = 4.469, p <.001). Das hybride Format bleibt also ohne Effekt auf das NE. Entgegen der Annahmen H3b und H3c besteht aber auch keine Wirkung auf einzelne NE-Dimensionen oder die 
Identifikation. Die Ergebnisse der t-Tests zeigen hier keine signifikanten Unterschiede zwischen $\mathrm{T} 1$ und $\mathrm{T} 2$.

Weitere Explorationen mit linearen multiplen Regressionsanalysen mit Identifikation und NE-Variablen als Kriterien und den Hintergrundfaktoren als Prädiktoren, die mit Identifikation oder NE-Variablen korrelierten, zeigten, dass Geschlecht und Involvement 7,3-27,8\% der Varianz bei Identifikation und NE-Dimensionen erklären: Frauen und Personen mit hohem Involvement haben höhere NE- und Identifikationswerte.

Untersuchungsfrage 4 analysierte mittels t-Tests mit einseitigem Signifikanztest, ob das synchron-hybride Inhaltsformat für höheren Widerstand gegen die persuasive Botschaft sorgt. Da explizite Informationen höheren Widerstand hervorrufen können im Vergleich zu rein narrativen, deren Botschaft implizit bleibt, wurde angenommen, dass das hybride Format zu höherer wahrgenommener Persuasionsabsicht ( $\mathrm{H} 4 \mathrm{a})$ und höherer Reaktanz (H4b) führt. Wider Erwarten zeigte das rein narrative Format (T1) mit mittlerer Effektstärke eine signifikant höhere wahrgenommene Persuasionsabsicht als das hybride $(\mathrm{T} 2), t(68)=2.299, p=.013, \Delta=-.55$, und auch marginal signifikant höhere Reaktanz, $t(68)=1.592, p=.058$.

Weitere explorative lineare multiple Regressionsanalysen mit schrittweisem Einschluss mit Reaktanz und wahrgenommener Persuasionsabsicht als Kriterien und Treatment sowie Variablen des personenbezogenen Hintergrunds, Outcomes zum Zeitpunkt zl («Voreinstellung»), Identifikation und NE als Prädiktoren zeigten, dass nicht nur das Inhaltsformat, sondern auch das Alter die Ausprägung der wahrgenommenen Persuasionsabsicht beeinflusst (je höher, desto mehr), und dass die Reaktanz neben Treatment auch von der Voreinstellung zu autoritativem Verhalten (je mehr diesem zugeneigt, desto geringer die Reaktanz), und Reaktanz sowie wahrgenommene Persuasionsabsicht v.a. vom Aufmerksamkeitsfokus (AF) beeinflusst werden: höheres AF geht mit weniger Reaktanz und Persuasionsabsicht einher.

Untersuchungsfrage 5 prüfte mittels t-Tests mit unabhängigen Stichproben, inwiefern sich die gedankliche Auseinandersetzung bei den Formaten unterscheidet. Weil es sich um verschiedene Verarbeitungsprozesse handelt, wurden Unterschiede für postrezeptive Reflexion (H5a) und rezeptionsbegleitende Elaboration $(\mathrm{H} 5 b)$ vermutet. Das Inhaltsformat 
beeinflusste aber weder Umfang noch Valenz der Reflexion (H5a) oder Elaboration (H5b). Insgesamt wurden zum Gegenstand deutlich mehr Gedanken notiert als zur Narration und mehr zustimmende - zur Geschichte waren es mehr kritische.

\subsection{Befunde zur Wirkweise II: Rolle der mutmasslich vermit- telnden Variablen}

Um die persuasive Wirkweise (noch Ziel 2) besser zu verstehen, suchten Untersuchungsfrage 6 - 7 zu klären, inwiefern die Variablen Rezeptionserleben, Identifikation, Widerstand und gedankliche Auseinandersetzung die persuasive Wirkung des Inhaltsformats vermitteln bzw. welche Rolle sie im Persuasionsprozess mit Informationen im synchron-hybriden Inhaltsformat spielen.

Untersuchungsfrage 6 überprüfte mit der SPSS-Erweiterung PROCESS (Preacher und Hayes 2008), ob mindestens ein Indikator von Identifikation, Rezeptionserleben und Widerstand die Wirkung des Inhaltsformats auf den Outcome mediiert (Baron und Kenny 1986) (H6), da diese Variablen in der Persuasionsliteratur als Mediator gehandelt werden. In den berechneten Mediationsmodellen war das Inhaltsformat die Prädiktorvariable (X), und Kriterien (Y) jene Outcome-Variablen, bei denen sich die zwei Treatmentgruppen signifikant unterschieden. Als Mediatoren (M) fungierten wahrgenommene Persuasionsabsicht (M1) und Identifikation (M2), da diese entweder mit X oder Y korrelieren. Es zeigten sich keine Mediationseffekte.

Zusätzliche explorative t-Tests mit hoher bzw. niedriger Identifikation, NE und Widerstand ermittelten, dass hohe Identifikation und hohe Werte der NE-Dimensionen Narrative Präsenz und Emotionales Engagement sowie geringe Aufmerksamkeit, hohe wahrgenommene Persuasionsabsicht und niedrige Reaktanz die Wirkung des Inhaltsformats auf mindestens eine Outcome-Variable vermittelten, wobei das hybride Format stets signifikant bessere Werte beim Persuasionsoutcome (Einstellungen und Verhaltensdeterminanten) erzielte als das narrative Format. In den übrigen Fällen gibt es bzgl. Persuasionswirkung keinen Unterschied zwischen den Formaten. Die gefundenen vermittelnden Effekte der Indikatoren von Widerstand, Rezeptionserleben und Identifikation jenseits der Mediatorrolle können hier demnach eher als moderierende Effekte verstanden werden. 
Untersuchungsfrage 7 suchte zu ergründen, inwieweit die Indikatoren von Rezeptionserleben, Identifikation, Widerstand und gedanklicher Auseinandersetzung bei den beiden Inhaltsformaten miteinander zusammenhängen und welche theoretischen Mechanismen belegt werden können. Tabelle 3 listet die Ergebnisse der Korrelationsanalysen.

\begin{tabular}{|c|c|c|c|c|c|c|c|c|c|c|}
\hline & \multicolumn{2}{|c|}{ Widerstand } & \multicolumn{3}{|c|}{ Identifikation } & \multicolumn{5}{|c|}{ Narrative Engagement } \\
\hline & Rea. & $\begin{array}{l}\text { Wg. } \\
\text { PA. }\end{array}$ & Ges. & Kind & Vater & Ges. & NU & AF & NP & $\mathrm{EE}$ \\
\hline \multicolumn{11}{|c|}{ Variablen Treatmentgruppe $\mathrm{T} 1(\mathrm{~N}=35)^{1}$} \\
\hline Reaktanz & 1.00 & $.67^{\star \star}$ & -.28 & -.09 & -.33 & $-.35^{\star}$ & -.07 & $-.47^{*}$ & -.17 & -.12 \\
\hline $\begin{array}{l}\text { Wg. Persuasions- } \\
\text { absicht }\end{array}$ & $.67^{\star \star}$ & 1.00 & 13 & .18 & .02 & -.08 & .31 & -.29 & .02 & .03 \\
\hline $\begin{array}{l}\text { Gedanken } \\
\text { Reflexion }\end{array}$ & -.02 & .08 & .25 & $.39^{\star}$ & .02 & .24 & .21 & .19 & .32 & .26 \\
\hline - Emotionen & -.15 & .03 & .22 & .28 & .09 & .01 & .25 & .03 & .13 & -.23 \\
\hline - ggst. & -.15 & -.02 & .22 & $.36^{\star}$ & -.01 & $.39 *$ & .25 & .27 & .29 & $.44^{\star}$ \\
\hline - gst. Positiv & -.08 & -.14 & -.11 & -.01 & -.10 & .02 & .01 & .01 & .01 & .17 \\
\hline $\begin{array}{l}\text { - gst. (tend.) } \\
\text { - positiv }\end{array}$ & -.24 & -.15 & .19 & $.34^{\star}$ & .05 & .25 & .12 & .26 & .23 & .21 \\
\hline $\begin{array}{l}\text { - gst. (tend.) } \\
\text { - negativ }\end{array}$ & -.01 & .08 & -.05 & .10 & -.26 & .17 & .20 & .12 & .08 & .17 \\
\hline $\begin{array}{l}\text { - narrationsbe- } \\
\text { zogen }\end{array}$ & $.34^{\star}$ & .13 & -.26 & -.28 & -.07 & $-.41^{*}$ & -.31 & -.31 & -.15 & -.33 \\
\hline $\begin{array}{l}\text { narrationsbe- } \\
\text { zogen negativ }\end{array}$ & $.43^{*}$ & .23 & -.22 & -.23 & -.10 & -.25 & -.17 & -.26 & -.14 & -.18 \\
\hline $\begin{array}{l}\text { Gedanken } \\
\text { Elaboration }\end{array}$ & -.10 & -.12 & .22 & .17 & .21 & .13 & .09 & .18 & .07 & .20 \\
\hline - gst. & -.23 & -.19 & .21 & .24 & .10 & .13 & .03 & .18 & .09 & .17 \\
\hline - gst. positiv & -.30 & -.20 & $.33^{\star}$ & .15 & .24 & .29 & .02 & .27 & .12 & $.38^{\star}$ \\
\hline $\begin{array}{l}\text { - narrationsbe- } \\
\text { zogen negativ }\end{array}$ & .16 & -.05 & -.11 & $-.37^{\star}$ & .11 & .01 & -.08 & .06 & -.04 & .02 \\
\hline
\end{tabular}

1 Anmerkung: $\mathrm{N}=70,{ }^{*} \mathrm{p}<.05$, kursiv gedruckt $=\mathrm{p}<.10$, Rea. $=$ Reaktanz, Wg. = Wahrgenommene, PA. = Persuasionsabsicht, Ges. = Gesamt, gst. = gegenstandsbezogen, tend. $=$ tendenziell. 


\begin{tabular}{|c|c|c|c|c|c|c|c|c|c|c|}
\hline & \multicolumn{2}{|c|}{ Widerstand } & \multicolumn{3}{|c|}{ Identifikation } & \multicolumn{5}{|c|}{ Narrative Engagement } \\
\hline & Rea. & $\begin{array}{l}\text { Wg. } \\
\text { PA. }\end{array}$ & Ges. & Kind & Vater & Ges. & NU & AF & NP & $\mathrm{EE}$ \\
\hline \multicolumn{11}{|c|}{ Variablen Treatmentgruppe $\mathrm{T} 2(\mathrm{~N}=35)$} \\
\hline Reaktanz & 1.00 & .27 & -.28 & -.19 & -.23 & -.02 & -.26 & -.25 & .06 & .10 \\
\hline $\begin{array}{l}\text { Wg. Persuasions- } \\
\text { absicht }\end{array}$ & .27 & 1.00 & .18 & .18 & .19 & .11 & .11 & -.33 & .17 & .24 \\
\hline $\begin{array}{l}\text { Gedanken } \\
\text { Reflexion }\end{array}$ & -.07 & -.01 & $.35^{\star}$ & .33 & $.39^{\star}$ & .27 & .19 & .33 & .16 & .12 \\
\hline - Emotionen & .07 & .10 & .26 & .09 & $.40^{\star}$ & .30 & .13 & .11 & .19 & .32 \\
\hline - gst. & .12 & -.14 & .19 & .27 & .25 & .17 & .16 & $.34^{\star}$ & .06 & -.01 \\
\hline - gst. positiv & .16 & .23 & .19 & $.34^{\star}$ & .17 & $.40^{\star}$ & $.36^{*}$ & .27 & .18 & $.37^{\star}$ \\
\hline $\begin{array}{l}\text { - gst. (tend.) } \\
\text { positiv }\end{array}$ & .13 & -.04 & .23 & .31 & .23 & .23 & .13 & $.38^{\star}$ & .03 & .13 \\
\hline $\begin{array}{l}\text { gst. (tend.) } \\
\text { negativ }\end{array}$ & $.39^{\star}$ & -.01 & -.30 & -.26 & -.18 & -.28 & -.16 & $-.36^{\star}$ & -.09 & -.15 \\
\hline $\begin{array}{l}\text { - narrationsbe- } \\
\text { zogen }\end{array}$ & -.16 & -.07 & .02 & -.07 & -.03 & .18 & .04 & -.05 & .25 & .16 \\
\hline $\begin{array}{l}\text { narrationsbe- } \\
\text { zogen negativ }\end{array}$ & -.08 & -.23 & -.09 & -.16 & -.09 & .16 & -.18 & .10 & .13 & .06 \\
\hline $\begin{array}{l}\text { Gedanken } \\
\text { Elaboration }\end{array}$ & -.18 & -.21 & .20 & .14 & .31 & .14 & .07 & .32 & .02 & .02 \\
\hline - gst. & -.18 & -.23 & .18 & .18 & .21 & .22 & .05 & $.38^{\star}$ & .12 & .50 \\
\hline - gst. positiv & .15 & -.07 & .08 & .06 & .05 & .26 & .06 & .11 & .26 & .19 \\
\hline $\begin{array}{l}\text { - narrationsbe- } \\
\text { zogen negativ }\end{array}$ & .18 & .18 & .01 & -.11 & .07 & .04 & .04 & -.09 & .05 & .06 \\
\hline \multicolumn{11}{|c|}{ Variablen Gruppen $\mathrm{T} 1$ und $\mathrm{T} 2(\mathrm{~N}=70)$} \\
\hline Reaktanz & 1.00 & $.49^{\star}$ & $-.28^{\star}$ & -.15 & $-.26^{\star}$ & -.19 & -.16 & $-.37^{\star}$ & -.07 & -.01 \\
\hline $\begin{array}{l}\text { Wg. Persuasions- } \\
\text { absicht }\end{array}$ & $.49^{\star}$ & 1.00 & .14 & .17 & .11 & -.00 & .23 & $-.33^{\star}$ & .05 & .12 \\
\hline $\begin{array}{l}\text { Gedanken } \\
\text { Reflexion }\end{array}$ & -.03 & -.02 & $.31^{\star}$ & $.35^{\star}$ & .17 & $.26^{\star}$ & .20 & $.27^{\star}$ & $.26^{\star}$ & .17 \\
\hline - gst. & -.03 & -.10 & .22 & $.31^{\star}$ & .09 & $.28^{\star}$ & .21 & $.31^{\star}$ & .19 & .21 \\
\hline - gst. positiv & .05 & .03 & .06 & .16 & .03 & .21 & .19 & .12 & .09 & $.27^{\star}$ \\
\hline $\begin{array}{l}\text { - gst. (tend.) } \\
\text { - positiv }\end{array}$ & -.06 & -.11 & .23 & $.31^{\star}$ & .14 & $.24^{\star}$ & .12 & $.32^{\star}$ & .14 & .16 \\
\hline $\begin{array}{l}\text { Gedanken } \\
\text { Elaboration }\end{array}$ & -.13 & -.17 & .23 & .15 & $.27^{\star}$ & .15 & .08 & $.26^{\star}$ & .06 & .10 \\
\hline - gst. & -.20 & -.22 & .22 & .21 & .16 & .18 & .02 & $.28^{\star}$ & .13 & .12 \\
\hline - gst. positiv & -.06 & -.12 & .20 & .10 & .16 & $.28^{\star}$ & .03 & .18 & .19 & $.29 *$ \\
\hline $\begin{array}{l}\text { - gst. (tend.) } \\
\text { - positiv }\end{array}$ & -.19 & -.15 & $.27^{\star}$ & $.27^{\star}$ & .21 & .16 & -.02 & .19 & .15 & .14 \\
\hline
\end{tabular}

Tab. 3.: Korrelationen (Spearman-Rho) ausgewählter Indikatoren der Informationsverarbeitung untereinander, nach Treatmentgruppe getrennt und beide Gruppen gemeinsam. 


\section{Interpretation und Diskussion}

\subsection{Zu Ziel 1: persuasive Wirksamkeit synchron-hybrider Inhaltsformate}

Eine wichtige Erkenntnis der Studie für die medienpädagogische Praxis der Gestaltung von Bildungsmaterialien ist, dass videobasierte EdutainmentAngebote mit synchron-hybridem Inhaltsformat, die also Geschichten und Erklärungen parallel darbieten, signifikant positiv auf Einstellungen und Verhaltensdeterminanten wirken und dass diese Wirkung die des rein narrativen Formats übersteigt. Rezipientinnen und Rezipienten des hybriden Formats bewerten das autoritative Zielverhalten und seine Folgen positiver und alternative Verhaltensweisen negativer (Einstellungsdeterminante). Sind gewisse Personenmerkmale gegeben, die den Einfluss des Materials vermitteln, z.B. dass Rezipientinnen und Rezipienten sich stärker mit den Figuren identifizieren können oder besser auf die Geschichte einlassen, sind sie ausserdem stärker zum Zielverhalten gewillt (Verhaltensintention), und glauben eher, es zu können (Kontrolldeterminante). Zudem zeigte sich, dass Personen mit schlechteren kognitiven Voraussetzungen (wenig Interesse am Thema, jung, unerfahren, geringe Aufmerksamkeit, ...) von diesem Format stärker profitieren. Allen übrigen ist das Format egal. Das hybride Format gleicht die Schwäche des narrativen Formats (die höhere Dekodierleistung wegen Unklarheit der Botschaft) offenbar aus und behält dabei dessen persuasive Vorteile. Das narrative Format scheint allein hinsichtlich des sozialen Drucks (Normdeterminante) wirksamer zu sein, unter der Bedingung, dass Rezipientinnen und Rezipienten schon vorher sehr positive Überzeugungen zum Zielverhalten oder eine hohe wahrgenommene Verhaltenskontrolle haben.

\subsection{Zu Ziel 2: persuasive Wirkweise synchron-hybrider Inhaltsformate}

Mit Blick auf die theoretische Weiterentwicklung der Persuasionsforschung kann aus den Befunden für die Wirkweise synchron-hybrider Inhaltsformate abgeleitet werden, dass das Capacity Modell (Fisch 2000) 
auch im Persuasionskontext zutrifft: narrative und argumentative Informationen können parallel und dabei komplementär verarbeitet werden mit signifikanten Vorteilen bei der Persuasionswirkung gegenüber dem rein narrativen Format. Für diese Hauptstärke der Tiefenverarbeitung scheinen mehrere Mechanismen und Wirkweisen der vermittelnden Faktoren bedeutsam, die den Erwartungen von Theorie und Empirie der Narrativen Persuasion z.T. widersprechen, aber wertvolle Hinweise für die Theorieentwicklung geben:

\subsubsection{Salienz als Persuasionsvorteil bei kognitiv ungünstigen Bedingungen}

Die höhere Salienz der persuasiven Botschaft im hybriden Format stellt in der Kombination mit narrativen Informationen einen Persuasionsvorteil dar, v.a. bei Rezipientinnen und Rezipienten mit geringer Aufmerksamkeit, Interesse und Fähigkeiten zur Auseinandersetzung mit den Inhalten. Mit Fisch (2000) wäre dieser Effekt u.a. damit zu erklären, dass hier mehr kognitive Kapazität auf die wichtigen persuasiven Informationen gelenkt wird.

\subsubsection{Förderung der Verarbeitung von narrativen sowie argumentativen Informationen}

Bei grosser Nähe der narrativen und argumentativen Inhalte sind NE und Identifikation im hybriden Format nicht beeinträchtigt, sondern begünstigen, wie in Modellen Narrativer Persuasion postuliert (u.a. Busselle und Bilandzic 2009, Moyer-Gusé 2008, Slater und Rouner 2002), die Informationsverarbeitung - beim hybriden Format aber nicht nur die der narrativen, sondern sogar der argumentativen Informationen. Faktoren wie Rezeptionserleben oder Identifikation können also, wie auch Moyer-Gusé und Nabi (2010) meinen, im Kontext rein narrativer Formate z.T. anders operieren als bei nicht-narrativen Informationen. Allerdings: adressieren die zusätzlichen Erklärungen nur eine Verhaltensdeterminante (hier die Einstellungskomponente), könnte die Fokussierung bei der Tiefenverarbeitung u.U. Nachteile bei anderen Aspekten (v.a. bei der sozialen Norm) zur 
Folge haben, wenn es zur Auslastung der kognitiven Ressourcen kommt. Hier sind weitere Untersuchungen angezeigt.

\subsubsection{Widerstandsreduktion als (mehrheitlicher) Vorteil des hybriden Formats}

Beim hybriden Format kommt es trotz höherer Salienz der persuasiven Botschaft zu geringerem Widerstand als beim rein narrativen Format, wobei der Aufmerksamkeitsfokus (AF) bei Reaktanz wie wahrgenommener Persuasionsabsicht eine besondere Rolle spielt. Insgesamt deuten sich unterschiedliche Mechanismen der Widerstandsreduktion an: Beim narrativen Format geht mit hoher Identifikation und NE (v.a. hohem AF) wie erwartet niedrige Reaktanz einher, beim hybriden wird mit steigendem AF stattdessen weniger Counterarguing geäussert, das hier zudem mit Reaktanz korreliert. Dies könnte daher rühren, dass die verarbeiteten argumentativen Informationen als kognitive Anker für die Gedankenproduktion fungieren. (Sick 2020) Dieser Befund stellt u.U. die Annahme in Frage, die Implizitheit der persuasiven Botschaft in Geschichten sei der Schlüssel zu geringerem Widerstand: interpretiert man die Befunde so, dass Rezipientinnen und Rezipienten des narrativen Formats ob der Implizitheit weniger Orientierung haben (zumal bei geringem AF) und stärker fürchten, unterschwellig beeinflusst zu werden, könnte hohes AF zu grösserer Klarheit verhelfen und so Widerstand senken; beim hybriden Format könnten die expliziten Informationen auch bei niedrigem AF diesen Effekt auffangen.

Allerdings erweist sich die Rolle des Widerstands in diesem Persuasionsprozess als komplexer: Die geringere wahrgenommene Persuasionsabsicht beim hybriden Format könnte sich u.U. negativ auf die wahrgenommene soziale Norm auswirken, mit evtl. schwächeren Persuasionseffekten, was in weiteren Studien zu prüfen wäre. 


\subsubsection{Keine Elaborationshemmung, sondern Förderung positi- ver Gedanken zum Objekt}

Entgegen der theoretischen Annahmen führt der narrative Verarbeitungsmodus nicht zu einer Reduktion der Elaboration, sondern geht mit mehr zustimmenden Gedanken zum Gegenstand einher, da Rezipientinnen und Rezipienten Sinn und Kongruenz zwischen der Welt der Narration und ihrer eigenen herstellen möchten (Hamby, Brindberg, und Daniloski 2017). Zwar unterscheiden sich das hybride und narrative Format nicht in Höhe und Valenz der gedanklichen Auseinandersetzung, aber es gibt durchaus Hinweise auf unterschiedliche Wirkmechanismen, z.B. bei NE: Beim hybriden Format sind stärker kognitiv orientierte NE-Dimensionen wichtig (mit höherem AF werden mehr positive Gedanken zum Gegenstand und weniger negative genannt), beim rein narrativen eher das emotionale Engagement - je höher, desto mehr (positive) Gedanken zum Gegenstand und weniger zur Story.

Für die medienpädagogische Bildungspraxis folgt aus diesen Erkenntnissen, dass das hybride Format bei Informationsmaterialen meist dem rein narrativen vorzuziehen ist, dass aber auch etliche weitere Faktoren jenseits des Inhaltsformats die Wirkung auf Einstellungen und andere Verhaltensdeterminanten beeinflussen. Für die theoretische Weiterentwicklung von Persuasionsmodellen bei Informationen mit synchron-hybridem Inhaltsformat ergeben sich aus der Studie einige wertvolle Hinweise, wobei auch weiterer Forschungsbedarf deutlich wurde, v.a. in Bezug auf die Rolle des Widerstands und der sozialen Norm bei der Persuasion mit synchronhybriden Formaten.

\section{Literatur}

Baron, Reuben M., und David A. Kenny. 1986. «The moderator-mediator variable distinction in social psychological research: Conceptual, strategic, and statistical considerations.» Journal of Personality and Social Psychology 51 (6): 11731182. https://doi.org/10.1037//0022-3514.51.6.1173.

Bilandzic, Helena, und Rick Busselle. 2013. «Narrative Persuasion.»In The Sage Handbook of Persuasion: Developments in Theory and Practice, hrsg. v. James P. Dillard \& Lijiang Shen, 200-219. Los Angeles: Sage.

Busselle, Rick, und Helena Bilandzic. 2009. «Measuring Narrative Engagement.» Media Psychology 12: 321-347. https://doi.org/10.1080/15213260903287259. 
Cacioppo, John T., und Richard E. Petty. 1984. «The elaboration likelihood model of persuasion.» Advances in Consumer Research 11 (1): 673-675.

Cohen, Jonathan. 2001. «Defining Identification: A Theoretical Look at the Identification of Audiences with Media Characters». Mass Communication and SocietY 4 (3): 245-264. https:// doi.org/10.1080/15213260903287259.

Dal Cin, Sonya, Mark P. Zanna, und Geoffrey T. Fong. 2004. «Narrative Persuasion and Overcoming Resistance.»In Resistance and Persuasion, hrsg. v. Eric S. Knowles \& Jay A. Linn, 175-191. Mahwah, NJ, US: Erlbaum.

De Graaf, Annecke, Hans Hoeken, José Sanders, und Johannes, W. J. Beentjes. 2012. «Identification as a Mechanism of Narrative Persuasion.» Communication Research 39 (6): 802-823. https://doi.org/10.1177/0093650211408594.

Dillard, James P., und Lijiam Shen. 2005. "On the Nature of Reactance and its Role in Persuasive Health Communication.» Communication Monographs 72: 144-168. https://doi.org/ 10.1080/03637750500111815.

Domke, Horst. 1991. «Erziehungsmethoden. Aspekte und Formen des Methodischen in der Erziehung.» Pädagogik. Eine Einführung, Bd. 2, hrsg. v. Erich Weber. Donauwörth: Auer.

Euler, Dieter und Angela Hahn. 2004. Wirtschaftsdidaktik. Berlin, Stuttgart, Wien: UTB.

Fernandes, Eulalia. 2016. «Resilience and emotional intelligence among adolescents as a function of perceived parenting style.» International Educational Scientific Research Journal 2 (7): 29-31.

Fisch, Shalom M.. 2000. "A Capacity Model of Children's Comprehension of Educational Content on Television.» Media Psychology 2 (1): 63-91. https://doi. org/10.1207/S1532785XM EPO2O1_4.

Fishbein, Martin. 2008. «A Reasoned Action Approach to Health Promotion.» Medical Decision Making 28 (6): 834-844. https://doi.org/10.1177/0272989X08326092.

Fishbein, Martin, und Isaac Ajzen. 1975. Belief, Attitude, Intention, and Behavior: An Introduction to Theory and Research. Reading, MA: Addison-Wesley.

Fishbein, Martin, und Isaac Ajzen. 2010. Predicting and Changing Behavior. The Reasoned Action Approach. New York: Psychology Press.

Graesser, Arthur C., Brent Olde, und Bianca Klettke. 2002. «How does the mind construct and represent stories?» In Narrative impact: Social and cognitive foundations», hrsg. v. Melanie C. Green, Jeffrey J. Strange, \& Timothy C. Brock, 229-262. Mahwah, NJ, US: Erlbaum.

Green, Melanie C., und Timothy C. Brock. 2000. «The Role of Transportation in the Persuasiveness of Public Narratives.» Journal of Personality and Social Psychology 79 (5): 701-721. https://doi.org/10.1037/0022-3514.79.5.701.

Green, Melanie C., und Timothy C. Brock. 2002. «In the Mind's Eye: Transportation-Imagery Model of Narrative Persuasion.» In Narrative impact: Social and cognitive foundations», hrsg. v. Melanie C. Green, Jeffrey J. Strange, \& Timothy C. Brock, 315-341. Mahwah, NJ, US: Erlbaum. 
Hamby, Anne, David Brinberg, und Kim Daniloski. 2017. «Reflecting on the journey: Mechanisms in narrative persuasion.» Journal of Consumer Psychology 27 (1): 11-22. https://doi. org/10.1016/j.jcps.2016.06.005.

Hoeken, Hans, und Karin M. Fikkers. 2014. «Issue-relevant thinking and identification as mechanisms of narrative persuasion.» Poetics 44, 84-99. https://doi. org/10.1016/j.poetic. 2014.05.001.

Krohne, Heinz W., Michael Hock. 2010. «Erziehungsstil.» In Handwörterbuch Pädagogische Psychologie (4., überarb. u. erw. Aufl.), hrsg. v. Detlef H. Rost, 159-167. Weinheim: Beltz.

Lang, Annie. 2000. «The Limited Capacity Model of Mediated Message Processing.» Journal of Communication 50 (1): 46-70. https://doi.org/10.1111/j.1460-2466.2000. tbo2833.x.

Moyer-Gusé, Emily. 2008. «Toward a theory of entertainment persuasion: Explaining the persuasive effects of entertainment-education messages.» Communication Theory 18 (3): 407-425. https://doi:10.1111/j.1468-2885.2008.00328.x.

Moyer-Gusé, Emily, Parul Jain und Adrienne H. Chung. 2012. "Reinforcement or Reactance? Examining the Effect of an Explicit Persuasive Appeal Following an Entertainment-Education Narrative.» Journal of Communication 62: 10101027. https://doi.org/10.1111/j.1460-2466.2012.01680.x

Moyer-Gusé, Emily, und Robin L. Nabi. 2010. «Explaining the effects of narrative in an entertainment television program: Overcoming resistance to persuasion.» Human Communication Research 36 (1): 26-52. https://doi.org/10.1111/ j.1468-2958.2009.01367.x.

Nell, Victor. 1988. Lost in a Book: The Psychology of Reading for Pleasure. Yale University Press, New Haven, CT.

Petty, Richard E.. 1997. «The Evolution of Theory and Research in Social Psychology: From Single to Multiple Effect and Process Models of Persuasion.»In The Message of Social Psychology: Perspectives on Mind in Society, hrsg. v. Craig McGarty \& S. Alexander Haslam, 268-290. Malden: Blackwell Publishing.

Petty, Richard E., und John T. Cacioppo. 1979. «Effects of Forwarning of Persuasive Intent and Involvement on Cognitive Responses and Persuasion.» Personality and Social Psychology Bulletin 5 (2): 173-176. https://doi. org/10.1177/014616727900500209.

Petty, Richard E., und John T. Cacioppo. 1986. Communication and Persuasion: Central and peripheral routes to attitude change. New York: Springer.

Petty, Richard E., John T. Cacioppo, Alan J. Strathman, und Joseph R. Priester. 2005. «To Think or not to Think: Exploring two Routes to Persuasion.»In Persuasion: Psychological Insights and Perspectives (2., überarb. Aufl.), hrsg. v. Timothy C. Brock \& Melanie C. Green, 81-116. Thousand Oaks, CA: Sage Publications.

Petty, Richard E., und Duane T. Wegener. 1999. «The Elaboration Likelihood Model: Current Satus and Controversies.» In Dual-Process Theories in Social PsYchology, hrsg. v. Shelly Chaiken \& Yaacov Trope, 41-72. New York/London: The Guilford Press. 
Preacher, Kristopher J., und Andrew F. Hayes. 2008. «Asymptotic and resampling strategies for assessing and comparing indirect effects in multiple mediator models.» Behavior Research Methods 40 (3): 879-891.

Schneewind, Klaus. 2008. Freiheit in Grenzen - Praktische Erziehungstipps. Eine DVD für Eltern von Kindern im Vorschulalter. München: Bayerisches Staatsministerium für Arbeit und Soziales, Familie und Integration.

Schneewind, Klaus. 1980. "Elterliche Erziehungsstile. Einige Anmerkungen zum Forschungsgegenstand. In Erziehungsstilforschung. Theorien, Modelle und Anwendung der Psycholo-gie elterlichen Erziehungsverhaltens, hrsg. v. Klaus Schneewind \& Theo Herrmann, 19-30. Bern: Huber.

Schneewind, Klaus. 2008. Freiheit in Grenzen - Praktische Erziehungstipps. Eine DVD für El-tern von Kindern im Vorschulalter. Bayerisches Staatsministerium für Arbeit und Soziales, Familie und Integration (Hrsg.), München.

Schneewind, Klaus, und Beate Böhmert. 2009. Kinder im Grundschulalter kompetent erziehen. Der interaktive Elterncoach "Freiheit in Grenzen" (2. überarb. Aufl.). Bern: Huber.

Shen, Fuyuan, Vivian C. Sheer, und Ruobing Li. 2015. "Impact of narratives on persuasion in health communication: A meta-analysis.» Journal of Advertising 44 (2), 105-113. https://doi. org/10.1080/00913367.2015.1018467.

Sick, Christina. 2020. «Überzeugen mit Geschichten und Argumenten. Die Wirkung videobasierter Narrationen mit parallelen Erklärungen auf Einstellungen und Verhaltensdeterminanten angehender Lehrkräfte zu Erziehungsstilen.» Dissertation, Pädagogische Hochschule Weingarten.

Sieland, Bernhard. 1994. "Erziehungsstil - Unterrichtsstil.» In Wörterbuch Schulpädagogik, hrsg. v. Rudolf W. Keck \& Uwe Sandfuchs, 99-101. Bad Heilbrunn: Julius Klinkhardt.

Slater, Michael D., und Donna Rouner. 2002. «Entertainment-education and elaboration likelihood: Understanding the processing of narrative persuasion.» Communication Theory 12 (2): 173-191. https://doi.org/10.1093/ct/12.2.173.

Steinberg, Laurence, Julie D. Elmen, und Nina S. Mounts. 1989. «Authoritative parenting, psychosocial maturity, and academic success among adolescents.» Child Development 60 (6): 1424-1436. https://doi.org/10.2307/1130932.

Studierendensekretariat PH Weingarten. 2016. «Studierendenstatistik Sommersemester 2016 (Erstellt am 25.05.2016).» Weingarten. Pädagogische Hochschule Weingarten. Zugriff 30.12.2018 http://www.ph-weingarten.de/filead$\mathrm{min} / \mathrm{redaktuer}$ /Homepage/Einrichtungen/Studierendensekretariat/Statistik/20161_Statistik.pdf.

Van Leeuwen, Lonneke, Bas van den Putte, Reint J. Renes, und Cess Leeuwis. 2017. «Do narrative engagement and recipients' thoughts explain the impact of an entertainment-education narrative on discouraging binge drinking?» Media Psychology 20 (2): 194-220. https:// doi.org/10.1080/15213269.2016.1142379. 